\title{
LA GENÈSE DE MA SCEUR L'ANGOISSE DE JAN ČEP
}

\author{
Jan Zatloukal
}

\begin{abstract}
My Sister anxiety, an autobiography of Jan Čep written first-hand in French, was the author's last attempt - fruitless - to gain the French public. It was published by the Christian Academy in Rome only after he died. Since it was issued in 1975 for the first time, some inaccuracies or even errors were spread over the origins of this posthumous autobiography of Čep. Thanks to a detailed research of the sources (an inedited diary, a type-written version of the French original), this paper rectifies these errors and helps to clarify the information on the genesis of My Sister anxiety, the structure of the manuscript and its dating, and the status of this piece of literature itself.
\end{abstract}

Keywords: genesis; dating; fragment; My Sister anxiety; Jan Čep; autobiography.

Résumé : Ma Sœur l'angoisse, l'ouvrage autobiographique que Jan Čep conçut directement en français, fut le dernier essai de l'auteur - infructueux - pour conquérir le public français. Elle ne fut publiée qu'après la mort de l'auteur en traduction tchèque par l'Académie chrétienne à Rome. Dès cette 1 ère édition en 1975 un certain nombre d'imprécisions, voire d'erreurs, s'étaient répandues autour de la genèse de cette autobiographie posthume. Grâce à l'étude détaillée des sources (journal inédit, version dactylographiée de l'original français) le présent article rectifie ces erreurs et contribue à préciser les informations s'attachant à la genèse de Ma Sœur l'angoisse, à la constitution du manuscrit et à sa datation, au statut même de l'ouvrage.

Mots clés : genèse ; datation ; fragment ; Ma Sœur l'angoisse ; Jan Čep ; autobiographie.

«L'angoisse est l'élément constant de ma vie, de mon expérience; c'est le mot qui vient le plus souvent sur mes lèvres; l'angoisse fidèle, bleue comme le ciel en plein été, vertigineux, mystérieux... ${ }^{1}$

Le manuscrit de Ma Sœur l'angoisse ${ }^{2}$ a attendu plus de dix longues années dans le tiroir de l'appartement rue Chanoinesse avant d'être finalement publié par l'Académie chrétienne de Rome en $1975 .{ }^{3}$ Son auteur reposait alors depuis plus d'un an dans le tombeau de la famille Du Bos à la Celle Saint-Cloud.

1 Journal, le 25 mars 1960.

2 En accord avec l'édition tchèque nous utilisons la forme du titre avec «a » minuscule : Ma Scur l'angoisse. Il est toutefois à noter que sur l'original du manuscrit dactylographié, le titre autographe est pourvu d'un « A » majuscule.

3 Ma Sœur l'angoisse fut publiée comme le 51 $1^{\mathrm{e}}$ volume de l'édition littéraire la Vigile. En 1964, du vivant de l'auteur, une sélection importante du manuscrit parut en français dans la revue Arena, n 20, p. 1-29. Le livre ne fut publié en République tchèque qu'après 1989 : Sestra úzkost, traduit du français par V. Čep et B. Fučík, Brno, Proglas, 1993 puis comme partie du VI volume des « Euvres complètes » Poutník na zemi (Un Pèlerin sur la 
Comme toute œuvre posthume (où l'auteur n'est plus en mesure de donner des explications sur son projet artistique ni de proposer des interprétations auctoriales), l'essai autobiographique de Čep recèle un nombre considérable de questions de toutes sortes dont les réponses, apportées, certes, souvent par de plus grands spécialistes, ne dissipent point l'ombre qui plane au-dessus. Ces réponses, concernant notamment la nature du projet auctorial et la genèse du livre, restent à ce jour partielles, plutôt hésitantes, et ce qui est plus grave, elles sont parfois tout à fait fausses. Depuis la première édition - nous l'appellerons romaine - un certain nombre d'idées reçues et même d'erreurs s'étaient constituées, répandues ensuite par le vent de la critique, et se virent reprises dans toutes les éditions ultérieures $(1993,1998)$ et tous les écrits portant sur la dernière œuvre de l'écrivain morave. La faute n'en incombe certainement à personne, sinon aux conditions malheureuses qui étaient celles d'un explorateur dépourvu de la quasi-totalité des matériaux dont il a absolument besoin pour son travail. Les éditeurs de l'édition romaine et les traducteurs du manuscrit français avaient hâte de présenter l'écrit inédit aux lecteurs tchèques, et surtout, ils ne disposaient pas de certains documents (correspondance, journal, etc.) dont nous avons su trouver le chemin. Par la suite, leurs erreurs initiales et certaines hypothèses erronées ne furent que reprises par des critiques littéraires et des chercheurs.

Ainsi donc allons-nous consacrer cette étude à rectifier et à préciser les informations s'attachant à la genèse de Ma Scur l'angoisse, à la constitution du manuscrit et à sa datation, au statut même de l'ouvrage.

Prenons comme point de départ la préface anonyme ${ }^{4}$ de l'édition romaine. C'est à partir d'elle que les imprécisions s'étaient transmises. Reprenons-en l'essentiel :

Ma Sour l'angoisse fut écrite en français et était destinée au public français. Pour cette raison sont donnés la perspective d'ensemble ainsi que certains détails explicatifs, non nécessaires au lecteur tchèque. L'omission volontaire des noms (effectuée avec une inconséquence apparente) s'inscrit dans ce projet. En effet, la plupart des noms n'auraient rien dit au lecteur français. Aussi, il est évident que Jan Čep, en écrivant ses souvenirs, ou plutôt sa confession, se précipitait et que le manuscrit supposait une rédaction finale.

Jan Čep écrivit Ma Sceur l'angoisse dans les années 1964-1965. Peu après, il subit une attaque cérébrale à la suite de laquelle sa main et sa jambe droites furent paralysées, aussi bien que, dans une large mesure, sa parole. C'est la raison pour laquelle il ne put faire d'un côté la version définitive du texte et de l'autre côté la version tchèque. Des amis et des spécialistes de l'œuvre de Čep se chargeaient de la traduction tchèque tout en étant conscients de ce qu'ils ne pouvaient, pas même de loin, atteindre l'exactitude de l'expression, la nuance mélodique et la richesse de langue de l'auteur. (Čep $1975: 5)^{5}$

Deux informations fausses ou au moins inexactes furent émises dans ce péritexte et docilement reprises et diffusées partout jusqu'à nos jours : la datation erronée et le caractère prétendument inachevé de Ma Sœur l'angoisse. Quasiment tous les comptes rendus postérieurs à l'édition romaine reprennent les années 1964-1965 comme la période pendant laquelle Čep écrivit son autobiographie, de même qu'ils soulignent sans exception l'écriture précipitée, la hâte d'un auteur qui sent déjà la mort approcher et qui s'applique à rédiger, à pas pressés, son testament. Ainsi, dans les comptes rendus de l'époque, des

terre), Brno, Proglas-Vyšehrad, 1998.

4 Quoique anonyme, on sait bien que le lot léonin incombait au frère de Jan Čep, Václav, et à l'ami proche et critique littéraire, Bedřich Fučík.

5 La traduction des citations tchèque est fournie par l'auteur de l'article. 
termes comme " énoncé non-fini » (nedořečená zpráva), « texte inachevé », " inachèvement », « caractère fragmentaire », etc. fourmillent. Antonín Kratochvil se montre le plus explicite à ce sujet, puisque nous pouvons lire chez lui des phrases telles que : "Chaque phrase laisse sentir la hâte de [vouloir] achever son épitaphe et son adieu littéraire. » Ou bien : «[...] la maladie impitoyable arracha la plume à Jan Čep achevant ses mémoires littéraires [...] En terminant les pages finales, il fut frappé par l'apoplexie. » (Kratochvil $1984: 7 ; 1976$ : 4). De telles phrases donnent l'effet d'une rupture brusque causée par la maladie ou la mort subites comme nous en rencontrons dans les journaux fictifs (plus précisément les romans écrits comme des journaux) où la dernière phrase reste inachevée. Songeons par exemple au Noud de vipères de F. Mauriac, au Journal d'un curé de campagne de G. Bernanos ou même aux Carnets de Jilji Klen de Čep lui-même. De tels propos, hyperboliques et pleins d'emphase, sont à prendre plus comme des jeux de style que comme des énoncés véridiques. Ils ne sont pas à être considérés comme fondés sur les faits, sinon on entre dans la fiction pure et simple : Čep tomba malade en été 1966 alors que, comme nous le verrons tout à l'heure, le manuscrit fut plus ou moins terminé trois ans auparavant.

Les mêmes informations se verront répétées dans les péritextes des deux éditions tchèques après 1989, même si en même temps, une certaine attitude réservée se laisse entrevoir notamment vis-à-vis de l'inachèvement de l'œuvre. L'auteur des épilogues et de notes d'édition, Mojmír Trávníček, n'oublie certes pas de souffler que Ma Sœur l'angoisse fut écrite en 1964-1965 et que la «plume lui [à Čep] fut arrachée de la main » mais en même temps il parsème son épilogue de 1993, de haute qualité d'ailleurs, de jugements plus nuancés à cet égard. Il ne parle plus d'une évidence de la hâte dans l'écriture mais seulement d'une " probabilité » ("Il est probable qu'elle fut écrite en hâte. » (Čep 1993 :123)) et l'affirmation du caractère non définitif de l'ouvrage revêt l'allure d'une hypothèse généralement reçue («On présume communément qu'il s'agit d'une œuvre qu'il ne fut donné pas à Čep de polir à sa forme définitive. » (Čep 1993 : 123)). Trávníček semble douter de la pérennité d'énoncés pareils et ne pas vouloir s'y impliquer personnellement. Il va jusqu'à se demander s'il ne s'agit pas là d'une «erreur et d'un malentendu » et rappelle que Čep employa pour plusieurs de ses proses l'indication générique de «fragment » ou d' « esquisse ». Comme nous le verrons tout à l'heure, cette interrogation va dans le bon sens.

Mais qu'est-ce qui nous permet de mettre en doute les propos des spécialistes les plus compétents en ce qui concerne l'œuvre de Jan Čep ? Qu'est-ce qui nous fait croire que nous détenons la vérité contre l'opinion généralement admise ? Comment pouvonsnous être tellement sûr de nous à propos de la genèse de Ma Sœur l'angoisse ? C'est que, grâce au journal personnel de Čep, nous avons pu reconstituer les différentes étapes de la genèse, reconstruire l'acte créateur depuis le projet initial jusqu'à la pointe finale du manuscrit. Nous allons maintenant rendre compte de nos observations en suivant et en commentant, pas à pas, la gestation douloureuse de Ma Sour l'angoisse.

La première mention, vague et incertaine, portant sur ce qui deviendra Ma Scur l'angoisse, est sans doute la notation dans le journal à la date du 21 avril 1960 : «Voudrais commencer l'autobiographie que m'a recommandé d'écrire Pierre Emmanuel il y a plus de deux ans. Mon enfance qui me paraît chaque jour [de] plus près. » (Journal inédit: le 21 avril 1960). Cette première mention, sans titre ni autres précisions du projet envisagé, révèle cependant des informations essentielles, et notamment qu'à l'origine de 
l'ouvrage se trouvait un stimulus venu de l'extérieur. Nous apprenons que l'essai autobiographique fut incité par le poète français et l'ami de Čep, Pierre Emmanuel, probablement dans une conversation ou un échange épistolaire privé quelque part au tournant de 1957/1958. ${ }^{6}$ En outre, c' est la présence de sa propre enfance qui s'annonce à son esprit avec de plus en plus d'insistance. Cependant, à ce moment, le projet ne demeure qu'en puissance, trahissant le désir profond de son auteur.

Quatre mois plus tard, deuxième mention, cette fois-ci pourvue directement d'un titre Ma Sour l'angoisse, quoique le titre en question soit débattu entre trois ouvrages différents, l'un déjà publié, le second étant en train d'être traduit, le troisième enfin marquant le projet autobiographique ${ }^{7}$ :

Ma Sœur l'angoisse. J'ai proposé ce titre à Demetz, pour le choix de mes proses qu'il est en train de traduire en allemand. Cela le fera tiquer sans doute ; cela lui paraîtra trop « subjectif », autobiographique. [...] J'avais déjà donné ce titre au choix de mes proses parues pendant la guerre. Et je le reprends pour le texte que j'ai commencé aujourd'hui, sans savoir encore trop quelle forme il prendra : essai autobiographique ou seulement une suite de méditations personnelles ? (Journal inédit : le 22 août 1960, termes soulignés par J. Z.)

C'est donc la date du 22 août 1960 qui est le commencement effectif de l'écriture ; le désir entre en acte. C'est aussi le moment de l'attribution du titre qui ne subira aucune modification au cours de la longue genèse du manuscrit. Si le titre paraît clair à l'auteur, le projet reste encore flottant, peu défini, plutôt intuitif que prémédité. C'est d'ailleurs répété une semaine plus tard lorsque Čep note dans son journal :

Je néglige un peu ce cahier depuis que j'ai commencé d'écrire dans le grand cahier bleu, mon « essai autobiographique » intitulé provisoirement « Ma Sœur l'angoisse ». Je ne sais pas ce que cela va donner, je ne me suis fait aucun plan. J'ai l'intention d'avancer au hasard, au fil de mes souvenirs, des expériences et des préoccupations majeures de ma vie. (Journal inédit : le 27 août 1960)

La dernière notation de cette année-là concernant le projet autobiographique, malgré la stabilisation du titre et même du sous-titre (" essai autobiographique »), confirme l'incertitude, sinon la perplexité devant l'entreprise à peine amorcée. Le 7 septembre, il avoue qu'il « n'y voi[t] toujours pas très clair » et que s'il « réusssi[t] à trouver [s]on chemin (une sorte de méthode) tout sera à réécrire ». (Journal inédit : le 7 septembre 1960)

Presque un an s'écoule sans qu'on puisse trouver une mention sur Ma Sœur l'angoisse dans le journal. Il ne faut cependant pas chercher très loin l'explication d'une lacune pareille. C'est sans doute qu'en dehors des vacances Čep manquait de loisirs pour avancer dans son autobiographie, étant submergé par ses obligations à la Radio. Ce n'est qu'en août 1961 que nous pouvons lire deux courtes notations au sujet du projet autobiographique. Les deux sont portées dans un esprit d'insatisfaction, voire presque de découragement. Il parle déjà d" " un demi-échec », insinue qu'il est "resté en panne avec [s]on essai autobiographique » et avoue que ce qu'il avait écrit jusque là ne le satisfait «qu'à moitié » (Journal inédit : le 23 et le 27 août 1961). Pour causes, il donne d'un côté la fatigue physique qui l'empêche de travailler systématiquement même dans les périodes

\footnotetext{
6 Les deux écrivains ont fait connaissance en 1948 à Prague, peu avant le putsch communiste. Le récit de cette rencontre ne manque d'ailleurs pas dans Ma Sœur l'angoisse.

7 En 1944 Sestra úzkost (La sœur angoisse) fut le titre d'un recueil de proses de Čep choisi et établi par Oldřich Králík pour les éditions Akord de Brno. En ce qui concerne la traduction allemande des nouvelles de Čep publiée en 1962 à Wien chez Herder Verlag, le volume porta finalement le titre différent de Zeit und Wiederkehr. Bilder aus Böhmen und Mähren. (Le temps et le retour. Images de Bohême et Moravie).
} 
d'une liberté relative, d'un autre côté le sentiment que « ce n'est pas cela » (Journal inédit : le 23 août 1961), trahissant clairement les embarras d'un auteur qui ne savait pas comment s'y prendre, où aller et comment continuer.

L'année suivante nous offre une seule remarque à propos de Ma Sœur l'angoisse, sans que le titre fût explicitement cité. A nouveau elle est faite au mois d'août, au cours des vacances en Italie que la famille Čep passa en compagnie de plusieurs amis tchèques dont l'abbé Heidler. D'après la notation, ce dernier avait incité le « projet d'une rédaction en commun » (Journal inédit : le 17 août 1962) des mémoires de Čep. Or, la conception de Heidler, présumant l'établissement d'un récit strictement chronologique et doté d'un appareil explicatif pour le public étranger, se montra en opposition radicale avec l'intention auctoriale. Au point d'aggraver encore l'incertitude de Čep au sujet de ses mémoires :

$\mathrm{Si}$ je suivais Dr. Heidler, il me faudrait réécrire mes textes d'une manière continue dans l'ordre chronologique, avec des explications à l'adresse des lecteurs peu cultivés, bref écrire un livre instructif et pédagogique. Il faudrait sacrifier les raccourcis qui s'enchaînent dans une logique intérieure, qui expriment une expérience directe, bien que souvent fragmentaire. Il me faudra renoncer, accepter définitivement mon destin, n'attendre aucune satisfaction personnelle de mon vivant ; il faudra continuer à vivre, penser, sentir et travailler pour ceux qui me liront peut-être après ma mort. (Journal inédit : le 17 août 1962)

À part l'accablement apparent de l'auteur, la citation nous révèle une information d'importance, à savoir qu'étant question de la rédaction, il est à peu près sûr que le texte devait toucher à sa fin.

Enfin le 27 août 1963, Čep annonce, sur un ton neutre dans son journal l'achèvement de l'autobiographie: "J'ai fini mon essai autobiographique par quelques méditations, par une sorte de prière. Je ne vois pas pour le moment ce que je pourrais ajouter de plus. » (Journal inédit : le 27 août 1963) Ceci est confirmé aussi par une rétrospection faite le 6 février 1964 où Čep fait remarquer qu'au cours des vacances de l'été précédent il avait fini sa "série d'essai[sic] autobiographique[sic], qui a pour titre 'Ma Sœur l'angoisse' » et qu'il " avai[t] commencée il y a presque quatre ans ». (Journal inédit : le 6 février 1964)

Résumons l'essentiel de ces passages parcourus dans le journal personnel de Čep. La période réelle pendant laquelle Čep écrivit Ma Sœur l'angoisse se situe entre août 1960 et 1963. Ceci dit, nous ne prétendons pas qu'il n'aura plus du tout touché au manuscrit. Il est tout à fait probable qu'au cours des années suivantes, il se soit décidé à retoucher certains passages, peut-être même à remanier légèrement l'ordre du récit dans la dernière section («Ici et là-bas »), notamment suite aux recommandations des lecteurs auxquels il a soumis l'ensemble du manuscrit. Mais, en aucun cas, il ne s'agit plus de remaniements substantiels, de corrections d'envergure comme par exemple l'ajout d'un chapitre, etc. L'ensemble du texte depuis le début jusqu'à la fin (série de quelques méditations achevée par une prière intime) fut déjà donné en 1963 comme le confirment non seulement la notation citée plus haut, mais également la publication d'extraits du manuscrit dans la revue Arena en 1964, reprenant déjà la totalité du récit, y compris la prière conclusive. Les années 1964-1965 indiquées communément comme la période de création ne sont plus dorénavant plausibles.

Une autre constatation porte sur la durée de la genèse de Ma Sœur l'angoisse. Il s'agit d'un manuscrit dont la gestation et la création furent les plus douloureuses et les plus longues de toute la carrière littéraire de Čep. Sans compter les petites retouches pro- 
bables (1964-66) l'écriture s'est étendue sur 3 ans, période qui dépasse au moins d'un an la durée de l'écriture du roman La Frontière de l'ombre (à peu près deux ans). L'écriture prolongée, sans doute à cause des conditions pour ainsi dire externes (manque de loisirs, fatigue) mais aussi par l'absence d'un projet prémédité et clairement défini. D'où aussi un caractère fragmentaire dû non seulement aux longues périodes de non-écriture (nous avons pu voir que Čep l'écrivait presque exclusivement pendant les mois de son congé) mais surtout à l'impossibilité, à la répugnance pour tout projet synthétique ou globalisant qui n'était pas dans la nature de l'écrivain Čep qui, même dans ses fictions, favorisait la composition fragmentaire. C'est dans cette perspective du fragmentaire délibéré et non de l'inachèvement qu'il faut appréhender aussi le sous-titre Fragments d'un essai autobiographique. C'est le fruit de l'écriture fondée sur le libre jeu de souvenirs et d'associations, sur cette « intention d'avancer au hasard » dont le résultat semblait aux lecteurs parfois peu satisfaisant par le manque d'équilibre dans la composition et par le décousu volontaire ${ }^{8}$. Il ne faut pas non plus oublier quelle était la conception de la mémoire humaine chez Čep. Celle-ci est faillible, regorge de trous, de lacunes, ne rend la vie que par fragments, par petites miettes. D'autant plus chez quelqu'un pour qui la vie humaine ne représente pas une suite cohérente de jours reliés par une logique de cause à effet, mais fait plutôt figure d" " un rêve sans consistance, un conte invraisemblable - un continent à demi submergé ou plutôt transfiguré et monté au ciel » (Čep 1975 : 24). Dans l'autobiographie qui est un genre basé sur la véracité des choses, on ne doit pas tricher. L'écrivain ne peut qu'humblement suivre sa mémoire lacunaire sans pouvoir recourir à l'affabulation. La meilleure preuve en est d'ailleurs donnée directement dans Ma Sœur l'angoisse. En amenant le lecteur à ses premiers souvenirs, l'auteur, dans un paragraphe transitoire (entre les éclairs de souvenirs les plus lointains et la description détaillée de l'univers de son enfance), explicite sa conception de la vie humaine en même temps qu'il laisse entrevoir quel sera son projet d'écriture :

Les souvenirs d'une vie ne peuvent constituer une histoire cohérente, même pas un ensemble d'histoires. La plupart du temps, ce ne sont que de courtes lueurs jetées par-ci par-là au gré des routes, comme des rayons de soleil dans l'herbe, sous les arbres, des fragments de visions ou de paroles, quelques gestes presque effacés, quelques échos de voix lointaines. Il faudrait en découvrir le sens et l'homogénéité secrète, les relier entre eux, trouver leur centre de gravité, leur point de convergence. Rien n'a été, sans doute, abandonné au hasard, mais rien non plus n'a été décidé d'avance... (Čep 1975 : 17-18)

Néanmoins, comme souvent, la réalité diffère ici sensiblement des propos déclarés. De toute façon, l'écriture de l'autobiographie ne se fondait pas uniquement sur des souvenirs flottants, sur une rêverie innocente, mais s'appuyait également sur le journal, c'est-à-dire une mémoire cultivée, fixée par écrit. Nous sommes donc témoins d'un procédé, finalement assez courant dans la littérature, qui consiste à reprendre des passages du journal personnel pour les insérer, d'une manière décalée, dans un récit autobiographique. Ces reprises sont repérables aussi chez Čep. Des passages du journal entrent dans le corps de Ma Scur l'angoisse souvent quasiment à l'identique, ne subissant que des transpositions

\footnotetext{
8 Dans une lettre à Mojmír Trávníček, Bedřich Fučík éreinte sévèrement Ma Scur l'angoise. Il lisait le manuscrit à Paris en 1966 au cours de sa visite chez l'infirme Čep et il avoue avoir du mal à lui cacher sa déception. Citons ses impressions, comme il les révèle à son ami Trávníček : «[F]ragment fait à la hâte, sans composition préméditée habituelle, fragment s'envolant au début de longue haleine et par rapport aux chapitres suivants tout à fait disproportionné et précipitant ensuite pêle-mêle aux diverses et simples mentions [...] ». (Hrabal 2003 : 187).
} 
stylistiques cosmétiques. Il est difficile de déterminer si et dans quels cas et mesure Čep utilisait son journal personnel en tant qu' « atelier d'écriture » et où il s'appuyait sur son journal pour simplement mieux se remémorer le passé. Ces ressemblances vont de la simple phrase jusqu' aux coupes de plusieurs alinéas, voire de pages entières. Ainsi, dans une notation de mars 1960 Čep, sortant d'une crise spirituelle après avoir traversé une période de sécheresse, s'efforce-t-il de vivifier et de reconquérir sa foi. Sa réflexion est de temps en temps coupée par une sorte de prière jaculatoire : "Seigneur, donnez-moi le sentiment de Votre présence! Vous êtes la voie, la vérité, et la vie ! (Journal inédit : le 11 mars 1960) Cette invocation du Seigneur, composée d'une imploration de sa présence et d'une citation de l'Evangile, est insérée sous une forme quelque peu élargie dans le dernier paragraphe de la prière clôturant Ma Scur l'angoisse :

Seigneur Jésus-Christ, qui êtes la voie, la vérité et la vie [...] Soyez-moi de plus en plus présent pendant cette dernière étape de ma route terrestre, et aidez-moi à être de plus en plus présent à Vous. (Čep 1975 : 148)

Ailleurs, ce sont de longues scènes entières qui entrent dans l'autobiographie, après avoir déjà été le sujet des rétrospections dans le journal. Il s'agissait, entre autres, du récit de l'agonie de son père, de plusieurs souvenirs de l'enfance, du bilan de son amour pour Jitka Fučíková, des passages d'introspection censés redresser l'équilibre de sa situation intérieure9.

Enfin, il reste à remarquer une particularité planant au-dessus de la dernière période de la création de Čep. En préparant les ouvrages d'exil pour l'édition samizdat dans les années 1980, Mojmír Trávníček s'aperçut que des passages entiers d'Un Pèlerin sur la terre (Rome, 1965) sont identiques aux sections finales de Ma Sœur l'angoisse consacrées aux années de l'exil. Ainsi observe-t-on une dialectique mystérieuse entre trois écrits distincts : journal intime, Un Pèlerin sur la terre - recueil de textes intimes choisis des méditations diffusées à Radio Free Europe et Ma Sœur l'angoisse - autobiographie posthume. Certains passages repris du journal sont « recyclés » dans les deux autres ouvrages, d'autres n'entrent que dans l'un ou l'autre. Si la section finale d'Un Pèlerin sur la terre est directement constituée de «fragments du journal » authentiques et non remaniés, dans Ma Sœur l'angoisse les mêmes notations journalières subissent, pour peu que ce soit, le travail de stylisation, le jeu de l'écriture. Quoi qu'il en soit, il est clair que le journal personnel servait à Čep de réservoir de sujets pour les textes à caractère intime, qu'il était susceptible de les reprendre à plusieurs reprises et de les réemployer dans les livres différents.

\section{Bibliographie}

ČEP, Jan, Journal, copie dactylographiée et inédite de l'original français, Paris: Institut des Etudes slaves.

ČEP, Jan, Ma sœur l'angoisse. Fragment d'un essai autobiographique, copie dactylographiée et inédite de l'original français, Paris : Institut des Etudes slaves.

ČEP, Jan (1964), Ma scur l'angoisse, Londres : Arena.

ČEP, Jan (1975), Sestra úzkost. Zlomky autobiografického eseje, Řím: Křest'anská akademie.

ČEP, Jan (1993), Sestra úzkost. Zlomky autobiografického eseje, Brno : Proglas.

\footnotetext{
9 Une fois, au début du chapitre final «Ici et là-bas », Čep cite explicitement un long extrait du journal, dans lequel, inspiré par la lecture d'une des nouvelles de sa jeunesse, il fait une analyse critique de sa création. (Journal inédit : le 22 octobre 1960)
} 
ČEP, Jan (1998), Poutník na zemi, Brno : Proglas-Vyšehrad.

Hrabal, Jiří (ed.) (2003), Listooni př́ležitosti. Dopisy Bedřicha Fučíka Mojmíru Trávníckovi, Olomouc: Aluze.

KRATOCHVIL, Antonín (1976), « Sestra úzkost. Na okraj posmrtného autobiografického eseje Jana Čepa ». In : Národní politika, nº 7, p. 4.

Kratochvil, Antonín (1984), «Deset let bez Jana Čepa ». In : České slovo, 30, n² 2, p. 7.

Jan Zatloukal

Katedra romanistiky

Filozofická fakulta UP

Kř́žkovského 10

77180 Olomouc

République tchèque

jan.zatloukal@upol.cz 